\title{
Locally Weyl invariant massless bosonic and fermionic spin-1/2 action in the $\left(\mathrm{W}_{\mathrm{n}(4)}, \mathrm{g}\right)$ and $\left(\mathbf{U}_{4}, \mathrm{~g}\right)$ space-times.
}

\author{
Agapitos Hatzinikitasit \\ University of Athens, \\ Nuclear and Particle Physics Division, \\ Panepistimioupoli GR-15771 Athens, Greece.
}

\begin{abstract}
We search for a real bosonic and fermionic action in four dimensions which both remain invariant under local Weyl transformations in the presence of non-metricity and contortion tensor. In the presence of the non-metricity tensor the investigation is extended to Weyl $\left(W_{n}, g\right)$ spacetime while when the torsion is encountered we are restricted to the Riemann-Cartan $\left(U_{4}, g\right)$ space-time. Our results hold for a subgroup of the Weyl-Cartan $\left(Y_{4}, g\right)$ space-time and we also calculate extra contributions to the conformal gravity.
\end{abstract}

KEYWORDS : Weyl invariance, Bosonic and fermionic actions, Contorsion, Non-metricity tensor.

\section{Introduction}

The notion of conformal invariance of microscopic phenomena is among the oldest and most intriguing ones in modern theoretical physics. Physically conformal invariance means that nothing depends on the choice of dimensional units such as lengths, etc. Conformally invariant field theories reveal some advantages ranging from classical equations of motion up to better quantum behaviour (the renormalization properties of matrix elements of the energy-momentum tensor are improved [1], quantum gravity is aymptotically free when a renormalization group analysis is performed [2]). Realistic gravity or unified field theory probably cannot be conformally invariant but many theories can stem from the spontaneously broken versions of invariant models.

In this work the non-metricity and torsion tensor are taken into account. The vanishing of torsion for the real world was Einstein's point of view that prevailed for a long time. This "superfluous" restriction seemed to add extra complications to the theory of General Relativity which turned out to have enormous success. On the other hand there was no compelling experimental reason to relax this condition.

In the case of Weyl's geometry one may achieve to make contact with Einstein gravity by resorting either to gauge fixing or to a dynamical symmetry breaking mechanism. The reduced space is Riemannian and the Weyl vector propagates as a massive spin-1 particle mediating the neutrino-neutrino interaction [3].

\footnotetext{
†e-mail: ahatzini@cc.uoa.gr
} 
The main theoretical advantage of gravity with torsion is that it links the spin of the matter fields with the space-time geometry [4]. Thus classical particles in the presence of torsion will follow different geodesics but there is no experimental measure to confirm such an effect. Phenomenological aspects of the torsion in the context of Standard Model were studied in [5]. These authors by implementing the propagating torsion into the abelian sector of the Standard Model were able to extract information about the torsion mass and torsion-fermion coupling by studying the four fermion contact interactions.

One could also be introduced to the exotic notion of torsion by the nonlinear sigma model action where it is represented locally on the field manifold as the curl of a second rank antisymmetric $B_{\mu \nu}$ [6] potential according to:

$$
S_{\mu \nu \lambda}=\partial_{[\mu} B_{\nu \lambda]}
$$

The present paper attempts to produce concrete expressions for conformally invariant, massless bosononic and fermionic spin-1/2 actions equipped with a general affine connection. To fulfil this task we organize our work in the following way.

Section 2 is devoted to a brief review of the background notions of the gravity with nonmetricity and torsion tensor. Our main concern is the decomposition of the contortion into irreducible representations of the proper orthochronous Lorentz group. This spliting will be proved to be extremely useful in the construction of the fermionic action. Also we state the well-known way the Christoffel symbol as well as the spin connection transform under local Weyl transformations.

In section 3 starting from the Einstein-Hilbert action with a cosmological constant in ndimensions and performing Weyl transformations to the determinant and the Ricci scalar of the space-time we end up with a massless bosonic action. We then proceed to incorporate initially the non-metricity tensor by constructing a conformally invariant action in $\left(W_{n}, g\right)$ space-time. To achieve this, a suitably defined conformally covariant derivative is needed. When we encounter the torsion it is possible to build an affine connection that leads to a conformally invariant action.

In section 4 using the decomposition of contortion, mentioned in section 2 , we write down the corresponding fermionic spin-1/2 conformal action for the two distinct cases of non-metricity and torsion tensor, in $\left(W_{4}, g\right)$ and $\left(U_{4}, g\right)$ space-times respectively. In both cases one can define once more conformally covariant derivates for fermions and convince itself that the tensor part of the torsion decouples from the fermionic action.

Section 5 enumerates and comments on which additional terms might contribute to the action. The selection is based on the conformal invariance of the free field theory and the restriction to quadratic fields and derivatives of $\mathcal{R}$ 's. These terms such as the Gauss-Bonnet theorem, the Pontrjagin and winding numbers are of topological nature. The Weyl tensor is also calculated and permits one to identify the terms introduced in the conformal gravity action in a rather natural way avoiding insertions by hand.

Finally, in the Appendix we establish all the necessary notation and identities employed in this paper. In the $\left(Y_{4}, g\right)$ space-time we write down the expressions giving the Riemann curvature, Ricci tensor and scalar as well as the corresponding quantities for the $\left(W_{n}, g\right)$ general case. 


\section{Background aspects of the gravity with torsion and non-metricity tensor.}

Any known conformally invariant field theory consists of two basic ingredients (see the review articles [7] and the huge amount of references therein). The first is the conformal transformation of the metric:

$$
\hat{g}_{\mu \nu}(x)=\Omega(x) g_{\mu \nu}(x)
$$

and the second the affine connection $\tilde{\Gamma}_{\nu \lambda}^{\mu}(g, T, M)$ which is defined by:

$$
\nabla_{\mu} g_{\nu \lambda}=N_{\mu \nu \lambda}
$$

As an exercise one can show that (3) leads to the most general form of the affine connection which reads t:

$$
\tilde{\Gamma}_{\nu \lambda}^{\mu}(g, T, M)=\Gamma_{\nu \lambda}^{\mu}(g)+A_{\nu \lambda}^{\mu}(T, M)
$$

$\Gamma_{\nu \lambda}^{\mu}(g)=\left\{\begin{array}{c}\mu \\ \nu \lambda\end{array}\right\}$ is the usual Christoffel symbol in the Riemann space-time $V_{4}, A_{\nu \lambda}^{\mu}=K^{\mu}{ }_{\nu \lambda}+$ $M_{\nu \lambda}^{\mu}$ with $K_{\nu \lambda}^{\mu}$ the so-called contortion tensor defined by

$$
\begin{aligned}
& K_{\mu \nu \lambda}=\frac{1}{2}\left(T_{\mu \nu \lambda} \cdot T_{\nu \mu \lambda} \cdot T_{\lambda \mu \nu} \cdot{ }^{\cdot \cdot}\right) \\
& =\frac{1}{2}\left(T_{\mu \nu \lambda} \ddot{*}+T_{\nu \lambda \mu} \ddot{*}+T_{\lambda \nu \mu}\right)
\end{aligned}
$$

and

$$
M_{\mu \nu \lambda}=\frac{1}{2}\left(N_{\mu \nu \lambda}-N_{\nu \mu \lambda}-N_{\lambda \mu \nu}\right)
$$

where $N_{\mu \nu \lambda}=w_{\mu} g_{\nu \lambda}+L_{\mu \nu \lambda}$ and the Cartan's torsion is defined in terms of the antisymmetric part of the affine connection as:

$$
T_{\nu \lambda}^{\mu \cdot \cdot}=2 \tilde{\Gamma}_{[\nu \lambda]}^{\mu}
$$

In contrast to $\Gamma_{\nu \lambda}^{\mu}(g)$, the torsion is a proper tensor under general coordinate transformations.

The tensor $K_{\mu \nu \lambda}$ has the following properties:

$$
\begin{aligned}
K_{\mu \nu \lambda} & =-K_{\nu \mu \lambda} \\
K_{\mu \nu}^{\mu}=0 ; \quad K_{\nu \mu}^{\mu} & =-K_{\nu \mu}^{\mu}=v_{\nu} ; \quad K_{\mu \nu \lambda}=\frac{1}{2} T_{\mu \nu \lambda}^{\cdots}
\end{aligned}
$$

while $M_{\mu \nu \lambda}$ satisfies:

$$
\begin{aligned}
M_{\mu \nu \lambda} & =M_{\mu(\nu \lambda)} \\
M^{\mu}{ }_{\mu \nu} & =-2 w_{\nu} \\
M_{\mu \nu}{ }^{\nu} & =w_{\mu}-L_{\nu \mu}{ }^{\nu}
\end{aligned}
$$

\footnotetext{
${ }^{1}$ Tilded and hatted tensors are refered to $\left(Y_{4}, g\right)$ space-time and their conformal equivalents respectively. The Riemann-Cartan $U_{4}$ space-time is a paracompact, Hausdorff, connected $C^{\infty}$ four dimensional manifold endowed with a locally Lorentzian metric and a linear affine connection obeying the metric compatibility condition: $\nabla_{\mu} g_{\nu \lambda}=\partial_{\mu} g_{\nu \lambda}-\Gamma_{\nu \mu}^{\rho} g_{\rho \lambda}-\Gamma_{\lambda \mu}^{\rho} g_{\rho \nu}=0$. In the present paper we will relax this restrictive condition to: $\nabla_{\mu} g_{\nu \lambda}=N_{\mu \nu \lambda}$ for which $d s^{2}=0$ is not preserved. One can require that $L_{\mu \nu \lambda}=L_{\mu(\nu \lambda)}$ and $g^{\nu \lambda} L_{\mu \nu \lambda}=0$. (instead one could equally make $L_{\mu \nu \lambda}$ completely symmetric). $L_{\mu \nu \lambda}$ is called the shear tensor since it stretches and shrinks length.

${ }^{2}$ Dots over the indices in (5) are reserved to keep track of their antisymmetry when needed.
} 
In (5) the last two terms belong, among others, to the symmetric part of the affine connection and the last property in (9) holds on the condition that the torsion is totally antisymmetric.

In four space-time dimensions the 24 independent components of torsion can be covariantly split into a traceless part and a trace: $T_{\mu \nu \lambda}=Z_{\mu \nu \lambda}+\frac{2}{3} g_{\mu[\nu} v_{\lambda]}$. In particular the decomposition into irreducible representations of the proper orthochronous Lorentz group $S O(3,1)$ is as follows [8, 9]:

1. the vector part $v_{\lambda}=g^{\mu \nu} T_{\mu \nu \lambda}$ of dimension 4 transforming as the $(1 / 2,1 / 2)$ ?,

2. the axial-vector part: $S_{\mu}=\frac{1}{6} \epsilon_{\mu \nu \lambda \rho} T^{\nu \lambda \rho}$, (or parity violating term) of the same dimension as before and corresponding to the Young tableau [111] and

3. the tensor $t_{\mu \nu \lambda}$ of mixed symmetry and dimension 16 f transforming according to $(3 / 2,1 / 2)$ $\oplus(1 / 2,3 / 2)$ and associated with the Young tableau [21]. It is expressed by:

$$
t_{\mu \nu \lambda}=T_{(\mu \nu) \lambda}-\frac{1}{3} g_{\mu \nu} v_{\lambda}+\frac{1}{3} g_{\lambda(\mu} v_{\nu)} .
$$

The tensorial part satisfies the following properties:

(a) is symmetric w.r.t. the first two indices:

$$
t_{\mu \nu \lambda}=t_{(\mu \nu) \lambda}
$$

(b) remains invariant under cyclic permutation of the indices:

$$
t_{\mu \nu \lambda}+t_{\lambda \mu \nu}+t_{\nu \lambda \mu}=0
$$

(c) and is traceless in the sense:

$$
g^{\mu \nu} t_{\mu \nu \lambda}=g^{\nu \lambda} t_{\mu \nu \lambda}=\epsilon^{\mu \nu \lambda \rho} t_{\nu \lambda \rho}=0 .
$$

Properties (14) and (16) are direct consequences of (13) while (15) holds in general for any Young tableau with the structure [21]. The torsion and contortion can be reexpressed in terms of the irreducible representations as:

$$
\begin{gathered}
T_{\mu \nu \lambda}=\frac{4}{3} t_{\mu[\nu \lambda]}+\frac{2}{3} g_{\mu[\nu} v_{\lambda]}+\epsilon_{\mu \nu \lambda \rho} S^{\rho}, \\
K_{\mu \nu \lambda}=-\frac{4}{3} t_{\lambda[\mu \nu]}+\frac{2}{3} g_{\lambda[\nu} v_{\mu]}+\frac{1}{2} \epsilon_{\mu \nu \lambda \rho} S^{\rho} .
\end{gathered}
$$

The conformal group $C_{\Omega}^{\infty}$ is infinite dimensional and the transformations of tensors (denoted by capital letters) for the subgroup of dilatations fall into two classes:

$$
\text { Class-I: } A \rightarrow \hat{A}=\Omega^{d(A)} A
$$

\footnotetext{
${ }^{3}$ The universal covering, i.e. the double covering, of $S O(3,1)$ is $S L(2, C)$ and its irreducible representations are parametrized by $\left(j_{1}, j_{2}\right)$ where $\mathbf{J}_{(i)}^{2} \rightarrow j_{i}\left(j_{i}+1\right), i=1,2$ [10].

${ }^{4}$ In the metric-affine space $\left(L_{d}, g\right)$ the irreducible representations have dimensions: $d, \frac{1}{6} d(d-1)(d-2)$ and $\frac{1}{3} d\left(d^{2}-4\right)$ respectively.
} 


$$
\text { Class-II: } B \rightarrow \hat{B}=F\left(\Omega^{d(B)}, \partial \ln \Omega\right)
$$

where $d(A)$ is the conformal weight (a real constant) of the quantity $A$ with suppressed indices. In the $V_{4}$ space-time the transformations:

$$
\begin{aligned}
g_{\mu \nu} & \rightarrow \hat{g}_{\mu \nu}=\Omega g_{\mu \nu} \\
e_{\mu}^{a} & \rightarrow \hat{e}_{\mu}^{a}=\Omega^{1 / 2} e_{\mu}^{a}
\end{aligned}
$$

determine uniquely the transformation law of the Christoffel symbol to be:

$$
\Gamma_{\nu \lambda}^{\mu} \rightarrow \hat{\Gamma}_{\nu \lambda}^{\mu}=\Gamma_{\nu \lambda}^{\mu}+\frac{1}{2}\left(\delta_{\nu}^{\mu} \partial_{\lambda} \ln \Omega+\delta_{\lambda}^{\mu} \partial_{\nu} \ln \Omega-g_{\nu \lambda} \partial^{\mu} \ln \Omega\right)
$$

where $\Omega(x)$ is a positively defined function. Both the explicit form and the transformation law of the spin connection are then deduced from the vierbein postulate:

$$
D_{\mu}(\Gamma, \omega) e_{\nu}^{a}(x)=\partial_{\mu} e_{\nu}^{a}-\Gamma_{\nu \mu}^{\lambda} e_{\lambda}^{a}+\omega_{\mu b}^{a} e_{\nu}^{b}=0
$$

and are:

$$
\begin{aligned}
\omega_{\mu a b} & =e_{b}^{\nu} \partial_{[\nu} e_{\mu] a}+e_{a}^{\rho} \partial_{[\mu} e_{\rho] b}+e_{a}^{\rho} e_{b}^{\nu} e_{\mu}^{j} \partial_{[\nu} e_{\rho] j} \\
& =\frac{1}{2}\left(\lambda_{b \mu a}+\lambda_{\mu a b}+\lambda_{b a \mu}\right) \\
\omega_{\mu b}^{a} \rightarrow \hat{\omega}_{\mu b}^{a} & =\omega_{\mu b}^{a}+\frac{1}{2} e_{\lambda}^{a}\left(e_{b}^{\nu} \delta_{\mu}^{\lambda} \partial_{\nu} \ln \Omega-e_{\mu b} \partial^{\lambda} \ln \Omega\right) .
\end{aligned}
$$

In the general case (contortion and non-metricity tensor are included) the transformation law of the spin connection will be determined in the same way as in the Riemann case namely:

$$
\tilde{\omega}_{\mu b}^{a}=\omega_{\mu b}^{a}+A_{\mu \nu}^{\lambda} e_{\lambda}^{a} e_{b}^{\nu} \rightarrow \hat{\tilde{\omega}}_{\mu b}^{a}=\hat{\omega}_{\mu b}^{a}+\hat{A}_{\mu \nu}^{\lambda} \hat{e}_{\lambda}^{a} \hat{e}_{b}^{\nu}
$$

where $\hat{\omega}_{\mu b}^{a}$ is given by (26) and the vierbein postulate is suitably defined.

\section{Local Weyl invariance of the bosonic action.}

In $n$-dimensions $(n \neq 2)$ the Einstein-Hilbert action with cosmological constant $\Lambda$ is:

$$
S_{E H}=\int d^{n} x \sqrt{g}\left(\frac{1}{G} R+\Lambda\right)
$$

where $g=-\operatorname{det} g_{\mu \nu}$ and $G$ is Newton's constant. Under the local conformal transformations:

$$
\begin{aligned}
\sqrt{g} & \rightarrow \sqrt{\hat{g}}=\Omega^{\frac{n}{2}} \sqrt{g} \\
R & \rightarrow \hat{R}=\Omega^{-1}\left[R-(n-1) D^{\mu} \partial_{\mu} \ln \Omega-\frac{1}{4}(n-1)(n-2) \partial^{\mu} \ln \Omega \partial_{\mu} \ln \Omega\right]
\end{aligned}
$$


it becomes:

$$
\begin{aligned}
& \hat{S}_{E H}=\int d^{n} x \sqrt{g} \Omega^{\frac{(n-2)}{2}} {\left[\frac{1}{G}\left(R-(n-1) D^{\mu} \partial_{\mu} \ln \Omega-\frac{1}{4}(n-1)(n-2) \partial^{\mu} \ln \Omega \partial_{\mu} \ln \Omega\right)\right.} \\
&\left.+\Lambda \Omega^{\frac{n}{2}}\right] .
\end{aligned}
$$

If one defines:

$$
\phi=\Omega^{\frac{n-2}{4}} \sqrt{\frac{2}{\xi G}}
$$

with $\xi$ a dimensionless constant, to be determined later on, and partially integrate (31) one arrives at:

$$
S_{E H}=\int d^{n} x \sqrt{g}\left[\frac{1}{2} g^{\mu \nu} \partial_{\mu} \phi \partial_{\nu} \phi+\frac{1}{2} \xi R \phi^{2}+\Lambda\left(\frac{\xi G}{2}\right)^{\frac{n}{n-2}} \phi^{\frac{2 n}{n-2}}\right] .
$$

The first term in brackets is the kinetic part of the real scalar field and the second for the distinguished value $\xi=\frac{1}{6}$ guarantees conformal invariance in the non-minimal coupling limit of $V_{4}$. As an exercise one can check that (33) is indeed invariant under conformal rescalings of the metric (2) provided that:

$$
\begin{aligned}
\hat{\phi} & =\Omega^{\frac{2-n}{4}} \phi \\
\xi & =\frac{1}{4}\left(\frac{n-2}{n-1}\right) .
\end{aligned}
$$

Let us now consider scalar fields of conformal weight $d(\phi)$ i.e.

$$
\hat{\phi}=\Omega^{d(\phi)} \phi
$$

then we have:

$$
\partial_{\mu} \hat{\phi}=\Omega^{d}\left(\partial_{\mu}+d(\phi) \partial_{\mu} \ln \Omega\right) \phi
$$

One can replace $\partial_{\mu}$ with the conformally covariant derivative $\mathcal{D}_{\mu}=\partial_{\mu}-d(\phi) w_{\mu}$ transforming as:

$$
\mathcal{D}_{\mu} \phi \rightarrow \Omega^{d(\phi)} \mathcal{D}_{\mu} \phi
$$

where $w_{\mu} \rightarrow w_{\mu}+\partial_{\mu} \ln \Omega$ and $d\left(\mathcal{D}_{\mu} \phi\right)=d(\phi)$. For convenience we distinguish and study the following two cases separately.

\section{Case I: Non-metricity tensor in $\left(\mathrm{W}_{\mathbf{n}}, \mathrm{g}\right)$ space-time.}

In the presence of non-metricity tensor we construct the following massless bosonic action in n-dimensions:

$$
S=\frac{1}{2} \int \sqrt{g}\left[g^{\mu \nu} \mathcal{D}_{\mu} \phi \mathcal{D}_{\nu} \phi+\xi \mathcal{R}(\tilde{\Gamma}) \phi^{2}+\Lambda\left(\frac{\xi G}{2}\right)^{\frac{n}{n-2}} \phi^{\frac{2 n}{n-2}}\right] d^{n} x .
$$

For the $\left(W_{n}, g\right)$ space-time our affine connection is given by:

$$
\tilde{\Gamma}_{\mu \nu}^{\lambda}=\Gamma_{\mu \nu}^{\lambda}+\frac{1}{2}\left(g_{\mu \nu} w^{\lambda}-\delta_{\nu}^{\lambda} w_{\mu}-\delta_{\mu}^{\lambda} w_{\nu}\right)
$$


Expression (40) enjoys the following properties:

$$
\begin{aligned}
\delta \tilde{\Gamma}_{\mu \nu}^{\lambda} & =0 \\
\tilde{\Gamma}_{\mu \nu}^{\lambda} & =\tilde{\Gamma}_{\nu \mu}^{\lambda} \\
\tilde{\Gamma}_{\mu \nu}^{\mu} & =\Gamma_{\mu \nu}^{\mu}-\frac{n}{2} w_{\mu} \\
g^{\mu \nu} \tilde{\Gamma}_{\mu \nu}^{\lambda} & =-\frac{1}{\sqrt{g}} \partial_{\mu}\left(\sqrt{g} g^{\mu \lambda}\right)+\left(\frac{n-2}{2}\right) w^{\lambda} .
\end{aligned}
$$

The first identity indicates the conformal invariance of the affine connection. The associated Ricci scalar has conformal weight $d(\mathcal{R}(\tilde{\Gamma}))=-1$ as one might check and the action is rewritten as:

$$
\begin{aligned}
S=\frac{1}{2} \int d^{n} x \sqrt{g} & {\left[g^{\mu \nu} \mathcal{D}_{\mu} \phi \mathcal{D}_{\nu} \phi+\xi\left(R+(n-1) D^{\mu} w_{\mu}-\frac{1}{4}(n-1)(n-2) w^{\mu} w_{\mu}\right) \phi^{2}\right.} \\
+ & \left.\Lambda\left(\frac{\xi G}{2}\right)^{\frac{n}{n-2}} \phi^{\frac{2 n}{n-2}}\right] .
\end{aligned}
$$

The action (45) is conformally invariant and when the Weyl vector field vanishes then $\mathcal{D}_{\mu} \phi=$ $\partial_{\mu} \phi$ recovering (33) in this limit.

One might wonder if there exist additional terms that contribute to (45) which do not break local conformal invariance. To answer this question we classify first all the building block tensors according to their conformal weight which ranges in the integer interval $[-1,2]$.

Table 1: Building block tensors versus their conformal weights

\begin{tabular}{|c|c|}
\hline Tensor & $d(A)$ \\
\hline \hline$\square, \mathcal{R}$ & -1 \\
\hline $\mathcal{R}_{\mu \nu}$ & 0 \\
\hline $\mathcal{H}_{\mu \nu}$ & 0 \\
\hline$g_{\mu \nu}, \mathcal{R}_{\mu \nu \lambda \rho}$ & 1 \\
\hline$\epsilon_{\mu \nu \lambda \rho}$ & 2 \\
\hline
\end{tabular}

In Table 1, $\square=g^{\mu \nu} \mathcal{D}_{\mu} \mathcal{D}_{\nu}$ and $\mathcal{H}_{\mu \nu}(w)$ is the curvature of the free $w_{\mu}$ Weyl field defined in close analogy to electromagnetism as: $\mathcal{H}_{\mu \nu}=\mathcal{R}_{\rho \mu \nu}^{\rho}=2 \mathcal{D}_{[\mu} w_{\nu]}=2 \partial_{[\mu} w_{\nu]}$. One might attempt to construct independent terms containing at most two $\mathcal{R}$ 's and $\mathcal{H}$ 's with total conformal weight -2 such as:

$$
\mathcal{R}_{\mu \nu} \mathcal{H}^{\mu \nu}, \quad g^{\mu \nu} \mathcal{R}_{\mu \nu \lambda \rho} \mathcal{R}^{\lambda \rho}, \quad \mathcal{R} \mathcal{R}_{\mu \nu \lambda \rho} \epsilon^{\mu \nu \lambda \rho}
$$

but further inspection reveals that all of them fall into the same class since they are proportional to $\mathcal{H}_{\mu \nu} \mathcal{H}^{\mu \nu}$. The issue of the valiable additional terms will be presented in a more detail in section 5 .

Case II: Contortion tensor in the $\left(\mathrm{U}_{4}, \mathrm{~g}\right)$ space-time. 
In the absence of the traceless part $t_{\mu \nu \lambda}=0$ of the contortion the associated affine connection reads:

$$
\tilde{\Gamma}_{\mu \nu}^{\lambda}=\Gamma_{\mu \nu}^{\lambda}+\frac{1}{3}\left(\delta_{\nu}^{\lambda} v_{\mu}-\delta_{\mu}^{\lambda} v_{\nu}+6 \epsilon_{\mu \nu}^{\lambda}{ }_{\alpha}^{\lambda} S^{\alpha}\right)
$$

which together with the scalar curvature:

$$
\mathcal{R}(K)=D^{\mu} v_{\mu}+\frac{1}{3} v^{2}+\frac{2}{3} S^{2}
$$

are no longer conformally invariant when the vector part $v_{\mu}$ transforms like the Weyl field $w_{\mu}$ and the axial vector part $S_{\mu}$ has conformal weight $d\left(S_{\mu}\right)=0$.

\section{Local Weyl invariance of the fermionic spin-1/2 action.}

Our main purpose in this section is by exploiting the conformal invariance of the fermionic action under the local Weyl rescalings:

$$
\begin{aligned}
e_{a}^{\mu} & \rightarrow \hat{e}_{a}^{\mu}=\Omega^{-1 / 2} e_{a}^{\mu} \\
e & \rightarrow \hat{e}=\Omega^{2} e \\
\Psi & \rightarrow \hat{\Psi}=\Omega^{-3 / 4} \Psi
\end{aligned}
$$

to build an action that possibly contains the irreducible fields $\left\{t_{\mu \nu \lambda}, v_{\mu}, S_{\mu}, N_{\mu \nu \lambda}\right\}$ and inherits conformal invariance.

For this reason we consider the most general f complex (Dirac) spin- $\frac{1}{2}$ fermion action in four dimensions which is given by:

$$
S_{1 / 2}=\int d^{4} x e^{\bar{\Psi} \not} \Psi
$$

where

$$
\not=e_{a}^{\mu} \gamma^{a}\left(\partial_{\mu}+\frac{1}{4} \tilde{\omega}_{\mu m n} \gamma^{m} \gamma^{n}\right)
$$

and

$$
\tilde{\omega}_{\mu m n} \gamma^{m} \gamma^{n}=\omega_{\mu m n} \gamma^{m n}+A_{\mu m n} \gamma^{m} \gamma^{n}
$$

In (53) $e_{\mu}^{a}$ is the vierbein for the metric $g_{\mu \nu}, e=\operatorname{det} e_{\mu}^{a}, \gamma^{a}$ are the $S O(4)$ Dirac matrices and $\gamma^{m n}=\frac{1}{2}\left[\gamma^{m}, \gamma^{n}\right]$.

\section{Case I: Non-metricity tensor in $\left(\mathbf{W}_{4}, \mathbf{g}\right)$ space-time.}

We consider first the case in which the "metric-compatibility" condition (under parallel transport in a Weyl space a vector changes its magnitude as well as the relative rotation) is satisfied. The vierbein postulate now becomes:

$$
\begin{array}{r}
\left(\mathcal{D}_{\mu}+d\left(e_{a}^{\nu}\right) w_{\mu}\right) e_{\nu}^{a}=0 \quad \text { or equivalently } \\
\nabla_{\mu} e_{\nu}^{a}=\partial_{\mu} e_{\nu}^{a}-\tilde{\Gamma}_{\mu \nu}^{\lambda} e_{\lambda}^{a}+\tilde{\omega}_{\mu}{ }^{a}{ }_{b} e_{\nu}^{b}=\frac{1}{2} w_{\mu} e_{\nu}^{a}
\end{array}
$$

\footnotetext{
${ }^{5}$ In principle one could also add a gauge connection $A_{\mu}=A_{\mu}^{\alpha} T_{\alpha}$ taking values in an arbitrary representation of an arbitrary Lie group but such a term is neglected at present but we will come to this point later.
} 
and the spin connection in terms of the vierbein is written as:

$$
\begin{aligned}
\tilde{\omega}_{\mu a b} & =\omega_{\mu a b}+{ }_{w} \omega_{\mu a b} \\
& =\frac{1}{2}\left(\lambda_{b \mu a}+\lambda_{\mu a b}+\lambda_{b a \mu}\right)+w^{\nu} e_{\nu[a} e_{b] \mu} .
\end{aligned}
$$

Plugging into (1/4) $\gamma^{\mu} \omega_{\mu m n} \gamma^{m} \gamma^{n}$ the expression (56) and making use of the identities (32) and (33) of the Appendix, a straightforward computation gives the action:

$$
S_{1 / 2}=\int d^{4} x e \bar{\Psi} e_{a}^{\mu} \gamma^{a}\left(D_{\mu}-\frac{3}{4} w_{\mu}\right) \Psi
$$

Similarly to the bosonic case one could also define the conformal derivative $\mathcal{D}_{\mu}$ acting on the fermion field as:

$$
\mathcal{D}_{\mu} \Psi=\left(D_{\mu}-d(\Psi) w_{\mu}\right) \Psi
$$

and then action (57) becomes invariant under local Weyl transformations. This can be proved provided that the Weyl field transforms in the usual way $w_{\mu} \rightarrow w_{\mu}+\partial_{\mu} \ln \Omega$ and bearing in mind that $\gamma^{\mu} \delta \omega_{\mu m n} \gamma^{m} \gamma^{n}=-(n-1) \gamma^{\mu} \partial_{\mu} \ln \Omega$. The conclusion driven from the above analysis is that the fermionic action remains intact, when the covariant derivative is replaced by its appropriate conformal partner, under local Weyl transformations and the non-metricity tensor is present. In other words in a Weyl space the Dirac spinor does not couple to the Weyl vector $w_{\mu}$ although this does not preclude the existence of other types of coupling terms [3].

\section{Case II: Contortion tensor in $\left(\mathrm{U}_{4}, \mathrm{~g}\right)$ space-time.}

Using (18) one could evaluate the term:

$$
\frac{1}{4} \gamma^{\mu} K_{\mu m n} \gamma^{m} \gamma^{n}=\frac{3}{4} \gamma^{\mu}\left(\frac{2}{3} v_{\mu}-\gamma_{5} S_{\mu}\right)
$$

It is worth noting that (59) is independent of the tensor $t_{\mu \nu \lambda}$ implying that the fermions are only coupled to the vector and axial-vector parts of the torsion.

The action then takes the form:

$$
S_{1 / 2}=\int d^{4} x e \bar{\Psi} e_{a}^{\mu} \gamma^{a}\left[D_{\mu}+\frac{1}{4}\left(2 v_{\mu}-3 \gamma_{5} S_{\mu}\right)\right] \Psi
$$

Assuming that $d\left(S_{\mu}\right)=0$ then apparently (60) can be written in an invariant way ( $v_{\mu}$ follows identical transformation law to that of the Weyl field) provided that $D_{\mu}$ is replaced by:

$$
\mathcal{D}_{\mu} \Psi=\left(D_{\mu}+\frac{2}{3} d(\Psi) v_{\mu}\right) \Psi
$$

From the final form of $S_{1 / 2}$ after performing the substitution (61) it is evident that the minimal Dirac action permits the spinor field to interact only with $S_{\mu}$ but not with $v_{\mu}$ and $t_{\mu \nu \lambda}$. 


\section{Additional contributions}

Let us now examine what extra independent terms could possibly contribute to (45) preserving conformal invariance of the free field theory and keeping at most quadratic terms in derivatives of fields and $\mathcal{R}$ 's.

1. A mass term $\sqrt{g} m_{w}^{2} w^{\mu} w_{\mu}$ will impose a fixed length scale in the theory and thus it is excluded since local Weyl invariance will be softly broken. The appearance of such a term is expected if one desires to make explicit contact with the classical Einstein gravity [3].

2. A nonlinear $-\frac{\lambda}{4 !} \sqrt{g} \phi^{4}$ potential does not break conformal invariance of the action but when we are interested in the free Langragian case it is neglected. An analogous quartic interaction with the scalar field $\phi$ replaced by $w_{\mu}$ does not pass the local Weyl transformation test as well.

3. On the ground of low energy physics higher derivative terms in (45) with respect to $w_{\mu}$ are disregarded. The kinetic term of the Weyl field is proportional to the square of the field strength and a term of the form $\left(\partial_{\mu} w^{\mu}\right)^{2}$ is abscent. This is explained by the fact that in a unitary vector theory both transversal and longitudinal components cannot propagate simultaneously [11].

4. The Euler form for an even dimensional manifold $\mathcal{M}$ is given by:

$$
\begin{aligned}
e_{2 l}(\mathcal{M}) & =\frac{(-1)^{l}}{(4 \pi)^{l} l !} \epsilon^{i_{1} i_{2} \cdots i_{2 l}} R_{i_{1} i_{2}} \wedge \cdots R_{i_{2 l-1} i_{2 l}} \\
& =\frac{(-1)^{l}}{(4 \pi)^{l} l !} I_{2 l} d V o l
\end{aligned}
$$

where $R^{i}{ }_{j}=\frac{1}{2} R^{i}{ }_{j k l} e_{\mu}^{k} e_{\nu}^{l} d x^{\mu} \wedge d x^{\nu}, I_{2 l}=2^{-l} \epsilon^{i_{1} i_{2} \cdots i_{2 l}} \epsilon^{j_{1} j_{2} \cdots j_{2 l}} R_{i_{1} i_{2} j_{1} j_{2}} \cdots R_{i_{2 l-1} i_{2 l} j_{2 l-1} j_{2 l}}$ and $d V o l=\sqrt{g} d^{2 l} x$ is the invariant volume element. Integration of $e_{2 l}(\mathcal{M})$ over a compact orientable Riemannian manifold provides the Gauss-Bonnet theorem expressed by:

$$
\chi_{2 l}(\mathcal{M})=\int_{\mathcal{M}_{2 l}} e_{2 l}(\mathcal{M})
$$

In four dimensions $I_{4}=R_{i j k l} R^{i j k l}-4 R_{i j} R^{i j}+R^{2}$ and the Gauss-Bonnet theorem in a coordinate basis becomes:

$$
\chi_{4}(\mathcal{M})=\frac{1}{32 \pi^{2}} \int_{\mathcal{M}_{4}} \sqrt{g}\left(R_{\mu \nu \lambda \rho} R^{\mu \nu \lambda \rho}-4 R_{\mu \nu} R^{\mu \nu}+R^{2}\right) d^{4} x
$$

The topological action (64) is also conformally invariant since $d\left(R_{\mu \nu \lambda \rho}\right)=1$ and $d\left(R_{\mu \nu}\right)=$ 0 .

5. Constructing the dualized tensor:

$$
{ }^{*} R_{\mu \nu \lambda \rho}=\frac{1}{2} \epsilon_{\mu \nu \sigma \tau} R_{\lambda \rho}^{\sigma \tau}
$$


then the Pontrjagin number in four dimensions reads:

$$
\begin{aligned}
\mathcal{P}_{4} & =\frac{1}{8 \pi^{2}} \int_{\mathcal{M}_{4}} R^{i j} \wedge R_{i j} \\
& =\frac{1}{16 \pi^{2}} \int_{\mathcal{M}_{4}} \sqrt{g}{ }^{*} \check{R}^{\mu \nu \lambda \rho} \check{R}_{\mu \nu \lambda \rho} d^{4} x
\end{aligned}
$$

This parity odd topological quantity is conformally invariant as one might check with the help of $d\left({ }^{*} R_{\mu \nu \lambda \rho}\right)=1$.

7. Another topological invariant is the winding number:

$$
N_{4}=\frac{1}{32 \pi^{2}} \int d^{4} x \sqrt{g}^{*} \mathcal{H}_{\mu \nu} \mathcal{H}^{\mu \nu}
$$

where ${ }^{*} \mathcal{H}_{\mu \nu}$ is the dualized field strength of the associated field.

8. When the torsion is present there is a topological invariant provided by the Nieh-Yan locally exact four form [12]:

$$
\begin{aligned}
N_{4} & =\int_{\mathcal{M}_{4}}\left(T^{i} \wedge T_{i}-R_{i j} \wedge \theta^{i} \wedge \theta^{j}\right) \\
& =\frac{1}{2} \int \sqrt{g}\left(\frac{1}{2} T_{\mu \nu}^{\sigma} T_{\sigma \lambda \rho}-R_{\mu \nu \lambda \rho}\right) \epsilon^{\mu \nu \lambda \rho} d^{4} x
\end{aligned}
$$

where $\theta^{i}=e_{\mu}^{i} d x^{\mu}$ is a non-coordinate basis. This invariant is not conformally inavariant since the N-Y form is a function of the local frame as opposed to the topological invariants discussed above.

9. The term $\sqrt{g} R^{2}$ is permissible and actually needed as a counterterm to the "two point" infinity. The term $\sqrt{g} \square R=\sqrt{g} g^{\mu \nu} \mathcal{D}_{\mu} \mathcal{D}_{\nu} R$ is also acceptable.

10. A Yakawa coupling of the form $\sqrt{g} \phi \bar{\Psi} \Psi$ is acceptable and generates mass to the fermions when present.

11. In $\left(W_{n}, g\right)(n \geq 3)$ space-time there is also the Weyl tensor which is conformally invariant and given by:

$$
\begin{aligned}
C_{\mu \alpha \beta \gamma}(\Gamma, M) & =R_{\mu \alpha \beta \gamma}(\Gamma)-\frac{1}{n-2}\left(g_{\mu \beta} R_{\alpha \gamma}+g_{\alpha \gamma} R_{\mu \beta}-g_{\mu \gamma} R_{\alpha \beta}-g_{\alpha \beta} R_{\mu \gamma}\right) \\
& -\frac{1}{(n-1)(n-2)} R(\Gamma)\left(g_{\mu \gamma} g_{\alpha \beta}-g_{\mu \beta} g_{\alpha \gamma}\right)+X_{\mu \alpha \beta \gamma}(\mathcal{H})
\end{aligned}
$$

where $g^{\alpha \gamma} C_{\mu \alpha \beta \gamma}=0$,

$$
X_{\mu \alpha \beta \gamma}(\mathcal{H})=\frac{1}{2(n-2)}\left(g_{\mu \beta} \mathcal{H}_{\alpha \gamma}+g_{\alpha \gamma} \mathcal{H}_{\mu \beta}-g_{\mu \gamma} \mathcal{H}_{\alpha \beta}-g_{\alpha \beta} \mathcal{H}_{\mu \gamma}\right)+\frac{1}{2} g_{\mu \alpha} \mathcal{H}_{\beta \gamma}(w)
$$

and

$$
\begin{aligned}
C_{\mu \alpha \beta \gamma}^{2}(\Gamma, M) & =R_{\mu \alpha \beta \gamma}^{2}(\Gamma)-\frac{4}{n-2} R_{\alpha \beta}^{2}(\Gamma)+\frac{2}{(n-1)(n-2)} R^{2}(\Gamma) \\
& +\frac{\left(n^{2}-2 n+4\right)}{4(n-2)} \mathcal{H}_{\alpha \beta}^{2} .
\end{aligned}
$$


So we observe from (71) that the field strength could be inserted into the gravity action in a natural way avoiding addition of such a term by force.

The situation is drastically changed if one moves on to a subgroup of the $\left(Y_{4}, g\right)$ space-time and considers the existence of torsion with only totally antisymmetric part:

$$
K_{\mu \nu \lambda}=\frac{1}{2} \epsilon_{\mu \nu \lambda \rho} S^{\rho}
$$

and $d\left(K_{\mu \nu \lambda}\right)=1$. A cumbersome but straightforward calculation gives the extra tensor:

$$
\begin{aligned}
X_{\mu \alpha \beta \gamma}(S) & =-\frac{1}{2}\left[g_{\mu[\beta} g_{\gamma] \alpha} S^{2}+2 \epsilon_{\mu \alpha \rho[\gamma} D_{\beta]} S^{\rho}+\left(g_{\mu[\beta} \epsilon_{\gamma] \rho \alpha \sigma}-g_{\alpha[\beta} \epsilon_{\gamma \rho \mu \sigma}\right)\right. \\
& \left.+\epsilon_{\mu \sigma \rho[\beta} \epsilon_{\gamma] \alpha \lambda}^{\sigma} S^{\rho} S^{\lambda}-\left(g_{\mu[\beta} S_{\gamma]} S_{\alpha}-g_{\alpha[\beta} S_{\gamma]} S_{\mu}\right)\right] .
\end{aligned}
$$

The term $\epsilon_{\mu \alpha \rho[\gamma} D_{\beta]} S^{\rho}$ in (73) spoils conformal invariance but one has the freedom to define the following conformally invariant tensor:

$$
Y_{\mu \alpha \beta \gamma}(S)=X_{\mu \alpha \beta \gamma}(S)+\epsilon_{\mu \alpha \rho[\gamma} D_{\beta]} S^{\rho} .
$$

and as a consequence the quadratic Weyl-like tensor

$$
\begin{aligned}
C_{\mu \alpha \beta \gamma}^{2}(\Gamma, M, S) & =R_{\mu \alpha \beta \gamma}^{2}(\Gamma)-\frac{1}{2} R_{\alpha \beta}^{2}(\Gamma)+\frac{1}{3} R^{2}(\Gamma) \\
& +\frac{3}{2}\left(\mathcal{H}_{\alpha \beta}^{2}(w)+\left(S^{2}\right)^{2}\right)-2 D^{\alpha} S^{\beta} \mathcal{H}_{\alpha \beta}(S)
\end{aligned}
$$

is conformally invariant. Notice here the appearance of the extra coupling terms $\mathcal{H}_{\mu \nu}^{2}(w)$, $\left(S^{2}\right)^{2}$ and $D^{\alpha} S^{\beta} \mathcal{H}_{\alpha \beta}(S)$.

12. Finally we address the possibility the Weyl strength $\mathcal{H}_{\mu \nu}$ to interact with the Yang-Mills field strength. In the non-Abelian case one could try the following coupling:

$$
\alpha \operatorname{tr}\left(\mathcal{H}^{\mu \nu} \mathcal{F}_{\mu \nu}\right)
$$

where $\mathcal{F}_{\mu \nu}=T^{a} F_{\mu \nu}^{a}, a=1, \cdots, \operatorname{dim}(\mathcal{G})$. The Langrangian (76) is locally scale invariant but not gauge invariant under the action of the symmetry group $\mathcal{G}$. The situation is improved in the Abelian case with electromagnetic field strength $F_{\mu \nu}(U(1)$ or F-curvature). Admissible candidates are:

$$
\beta_{1} \mathcal{H}^{\mu \nu} F_{\mu \nu} ; \quad \beta_{2} \epsilon^{\mu \nu \rho \sigma} \mathcal{H}_{\mu \nu} F_{\rho \sigma}
$$

with $d\left(A_{\mu}\right)=d\left(F_{\mu \nu}\right)=0$. One might convince itself that these types of interactions are simultaneously locally Weyl and $U(1)$ invariant. 


\section{Conclusions}

The lessons this exercise taught us are summarized in the following:

A. The Einstein-Hilbert action can be conformally reduced to a massless bosonic action in n-dimensions and the non-metricity and torsion tensor can be included in a conformally invariant way respectively. This is achieved by defining the conformal partner of the covariant derivative and adjusting the affine connection in the case of torsion.

B. A conformally invariant fermionic action for the spin- $1 / 2$ field is also constructed for the two cases under consideration. Applying the idea of conformal derivative we write down the corresponding actions and show that the vector and tensorial parts of the Cartan's torsion do not couple minimally to fermions.

C. There are certain topological invariants such as the Euler characteristic, the Pontrjagin and winding numbers that contribute to the action without affecting the conformal properties of the theory. In a subgroup of the $\left(Y_{4}, g\right)$ space-time one could also construct a Weyl-like tensor the square of which provides new terms to the gravity action such as $\mathcal{H}_{\mu \nu}^{2}(w),\left(S^{2}\right)^{2}$ and $D^{\alpha} S^{\beta} \mathcal{H}_{\alpha \beta}(S)$. We also examine the possibility of coupling the photons to the Weyl vector.

\section{Acknowledgements}

I am grateful to F. W. Hehl for suggesting some crucial improvements to the manuscript and also to thank T. Christodoulaki and G. Diamandi for valuable discussions.

\section{Appendix}

Throughout this paper flat (tangent space) indices are denoted by Latin letters subjected to local Lorentz rotations and boosts. Greek characters stand for curved indices and are only subjected to local translations. Roman indices may be raised using the flat-space metric $\eta^{a b}$; and Greek indices may be raised or lowered using:

$$
g^{\mu \nu}(x)=\eta^{a b} e_{a}^{\mu}(x) e_{b}^{\nu}(x) .
$$

Anti-symmmetrisations carry a weight of $1 / n$ ! where $n$ is the number of indices involved in these operations.

The symbols we reserve to denote different types of covariant derivatives are:

$D_{\mu} \quad$ for covariant derivative based on the Christoffel symbol,

$\mathcal{D}_{\mu} \quad$ for conformal convariant derivative and

$\nabla_{\mu} \quad$ for covariant derivative built on an affine connection

The covariant derivative of a tensor with q-contravariant and p-covariant indices $(p>1)$ is defined as:

$$
D_{\mu} T_{\beta_{1} \beta_{2} \cdots \beta_{p}}^{\alpha_{1} \alpha_{2} \cdots \alpha_{q}}=\partial_{\mu} T_{\beta_{1} \beta_{2} \cdots \beta_{p}}^{\alpha_{1} \alpha_{2} \cdots \alpha_{q}}+\left[\Gamma_{\mu}, T\right]_{\beta_{1} \beta_{2} \cdots \beta_{p}}^{\alpha_{1} \alpha_{2} \cdots \alpha_{q}}
$$


where $\left[\Gamma_{\mu}, T\right]_{\beta_{1} \beta_{2} \cdots \beta_{p}}^{\alpha_{1} \alpha_{2} \cdots \alpha_{q}}=\Gamma_{\mu \nu}{ }^{\alpha_{1}} T_{\beta_{1} \beta_{2} \cdots \beta_{p}}^{\nu \alpha_{2} \cdots \alpha_{q}}+\cdots+\Gamma_{\mu \nu}{ }^{\alpha_{q}} T_{\beta_{1} \beta_{2} \cdots \beta_{p}}^{\alpha_{1} \alpha_{2} \cdots \nu}-(-1)^{p} \Gamma_{\mu \beta_{1}}{ }^{\nu} T_{\nu \beta_{2} \cdots \beta_{p}}^{\alpha_{1} \alpha_{2} \cdots \alpha_{q}}-\cdots-$ $-(-1)^{p} \Gamma_{\mu \beta_{p}}^{\nu} T_{\beta_{1} \beta_{2} \cdots \nu}^{\alpha_{1} \alpha_{2} \cdots \alpha_{q}}, p \geq 2$ and $\Gamma_{\mu \nu}^{\lambda}=\left\{\begin{array}{c}\lambda \\ \mu \nu\end{array}\right\}=\frac{1}{2} g^{\lambda \rho}\left(\partial_{\mu} g_{\nu \rho}+\partial_{\nu} g_{\mu \rho}-\partial_{\rho} g_{\mu \nu}\right)$ is the usual Christoffel symbol. The commutation relation of the covariant derivatives is:

$$
\left[\nabla_{\mu}, \nabla_{\nu}\right] V_{\lambda}=-R_{\mu \nu \lambda}^{\rho} V_{\rho}-\left[2 K_{\mu \nu}^{\rho}+N_{\mu \nu}^{\rho}-N_{\nu \mu}^{\rho}\right] \nabla_{\rho} V_{\lambda}
$$

One could also define a conformally covariant derivative for a tensor of the general type $T_{\beta_{1} \beta_{2} \cdots \beta_{p}}^{\alpha_{1} \alpha_{2} \cdots \alpha_{q}}$ having conformal weight $d\left(T_{\beta_{1} \beta_{2} \cdots \beta_{p}}^{\alpha_{1} \alpha_{2} \cdots \alpha_{q}}\right)=d(T)$ as follows:

$$
\mathcal{D}_{\mu} T_{\beta_{1} \beta_{2} \cdots \beta_{p}}^{\alpha_{1} \alpha_{2} \cdots \alpha_{q}}=\nabla_{\mu} T_{\beta_{1} \beta_{2} \cdots \beta_{p}}^{\alpha_{1} \alpha_{2} \cdots \alpha_{q}}-d(T) w_{\mu} T_{\beta_{1} \beta_{2} \cdots \beta_{p}}^{\alpha_{1} \alpha_{2} \cdots \alpha_{q}}
$$

where $w_{\mu}$ is the Weyl field. Thus for example the conformal derivative of scalar field is: $\mathcal{D}_{\mu} \phi=$ $\partial_{\mu}-d(\phi) w_{\mu} \phi$ while for the metricity tensor $g_{\mu \nu}$ with conformal weight $d\left(g_{\mu \nu}\right)=1$ holds: $\mathcal{D}_{\mu} g_{\nu \lambda}=0$. The covariant derivative of a vector $A_{\mu}$ is given by:

$$
\nabla^{\mu} A_{\mu}=D^{\mu} A_{\mu}-A_{\mu} w^{\mu}=\nabla_{\mu} A^{\mu}+A_{\mu} w^{\mu} .
$$

The commutation of the conformal derivatives gives:

$$
\left[\mathcal{D}_{\mu}, \mathcal{D}_{\nu}\right] A_{\lambda}=-\left(R_{\mu \nu \lambda}^{\rho} A_{\rho}+d\left(A_{\lambda}\right) \mathcal{H}_{\mu \nu} A_{\lambda}\right)
$$

The Riemann curvature, Ricci tensor and the scalar curvature are defined by:

$$
\begin{aligned}
R_{\rho \sigma \nu}^{\mu} & =\partial_{\rho} \Gamma_{\sigma \nu}^{\mu}+\Gamma_{\rho \alpha}^{\mu} \Gamma_{\sigma \nu}^{\alpha}-\rho \leftrightarrow \sigma \\
R_{\rho \nu} & =R_{\rho \mu \nu}^{\mu} \\
R & =g^{\mu \nu} R_{\mu \nu}
\end{aligned}
$$

and in the presence of torsion and non-metricity tensors (with $L_{\mu \nu \lambda} \neq 0$ ) are given by:

$$
\begin{aligned}
\mathcal{R}_{\nu \lambda \rho}^{\mu}(\Gamma, A) & =\mathcal{R}_{\nu \lambda \rho}^{\mu}(\Gamma, M)+\mathcal{R}_{\nu \lambda \rho}^{\mu}(K)-w^{\sigma} K_{\sigma[\rho}^{\mu} \delta_{\lambda]}^{\nu}+w^{\mu} K_{[\rho}^{\sigma \nu} g_{\lambda] \sigma}-w_{\sigma} K_{[\rho}^{\sigma \nu} \delta_{\lambda]}^{\mu} \\
& -w^{\nu} K_{[\rho \lambda]}^{\mu}-K_{\sigma[\rho}^{\mu} \mathcal{P}_{\lambda] \nu}^{\sigma}-K_{\nu[\rho}^{\sigma} \mathcal{P}_{\lambda] \sigma}^{\mu} \\
\mathcal{R}_{\nu \rho}(\Gamma, A) & =\mathcal{R}_{\nu \rho}(\Gamma, M)+\mathcal{R}_{\nu \rho}(K) \\
& -\frac{1}{2}\left[g_{\nu \rho} w^{\mu} v_{\mu}-v_{\rho} w_{\nu}+w^{\mu}\left(2 K_{\mu \nu \rho}+K_{\nu \mu \rho}-K_{\rho \nu \mu}\right)\right] \\
& +\frac{1}{2}\left[v_{\sigma} \mathcal{P}_{\rho \nu}^{\sigma}+K_{\sigma \rho}^{\mu} \mathcal{P}_{\mu \nu}^{\sigma}-K_{\nu \mu}^{\sigma} \mathcal{P}_{\rho \sigma}^{\mu}\right] \\
\mathcal{R}(\Gamma, A)=\mathcal{R}(\Gamma, M)+ & \mathcal{R}(K)-\frac{1}{2}\left[5 w^{\mu} v_{\mu}-v^{2}+v_{\mu} \mathcal{P}_{\nu}^{\mu \nu}+K_{\sigma \nu}^{\mu}\left(\mathcal{P}_{\mu}^{\sigma \nu}-\mathcal{P}_{\mu}^{\nu \sigma}\right)\right]
\end{aligned}
$$

where:

$$
\begin{aligned}
\mathcal{R}_{\nu \lambda \rho}^{\mu}(\Gamma, M) & =R_{\nu \lambda \rho}^{\mu}(\Gamma)-g_{\nu[\lambda} D_{\rho]} w^{\mu}+\delta_{\nu}^{\mu} D_{[\rho} w_{\lambda]}+\delta_{[\lambda}^{\mu} D_{\rho]} w_{\nu} \\
& -\frac{1}{2}\left[g_{\nu[\lambda} w_{\rho]} w^{\mu}+\delta_{[\lambda}^{\mu} g_{\rho] \nu} w^{2}+\delta_{[\rho}^{\mu} w_{\lambda]} w_{\nu}\right] \\
& -D_{[\rho} \mathcal{P}_{\lambda] \nu}^{\mu}-\frac{1}{4} w^{\mu}\left(\mathcal{P}_{\rho \nu \lambda}-\mathcal{P}_{\lambda \nu \rho}\right) \\
& -\frac{1}{2}\left[w_{\sigma} \delta_{[\lambda}^{\mu} \mathcal{P}_{\rho] \nu}^{\sigma}+w^{\sigma} g_{\nu[\lambda} \mathcal{P}_{\rho] \sigma}^{\mu}+\mathcal{P}_{\sigma[\rho}^{\mu} \mathcal{P}_{\lambda] \nu}^{\sigma}\right]
\end{aligned}
$$




$$
\begin{gathered}
\mathcal{R}_{\nu \rho}(\Gamma, M)=R_{\nu \rho}+\frac{1}{2}\left(g_{\nu \rho} D_{\mu} w^{\mu}+3 D_{\rho} w_{\nu}-D_{\nu} w_{\rho}-g_{\nu \rho} w^{2}+w_{\nu} w_{\rho}\right) \\
+\frac{1}{2} D_{\mu} \mathcal{P}_{\rho \nu}^{\mu}-\frac{1}{4} w^{\mu}\left(2 \mathcal{P}_{\mu \rho \nu}+\mathcal{P}_{\rho \nu \mu}+\mathcal{P}_{\nu \rho \mu}\right)-\frac{1}{4} \mathcal{P}_{\sigma \rho}^{\mu} \mathcal{P}_{\mu \nu}^{\sigma} \\
\mathcal{R}(\Gamma, M)=R+3 D_{\mu} w^{\mu}-\frac{3}{2} w^{2}+\frac{1}{2} D_{\mu} \mathcal{P}_{\nu}^{\mu \nu}-\frac{1}{4}\left(2 w^{\mu} \mathcal{P}_{\mu \nu}{ }^{\nu}+\mathcal{P}_{\sigma \nu}^{\mu} \mathcal{P}_{\mu \nu}^{\sigma \nu}\right) \\
\mathcal{R}_{\nu \lambda \rho}^{\mu}(K)=D_{\lambda} K_{\nu \rho}^{\mu}-D_{\rho} K_{\nu \lambda}^{\mu}-K_{\sigma \rho}^{\mu} K_{\nu \lambda}^{\sigma}+K_{\sigma \lambda}^{\mu} K_{\nu \rho}^{\sigma} \\
\mathcal{R}_{\nu \rho}(K)=D^{\mu} K_{\mu \nu \rho}+D_{\rho} v_{\nu}-v_{\sigma} K_{\nu \rho}^{\sigma}-K_{\sigma \rho}^{\mu} K_{\nu \mu}^{\sigma} \\
\mathcal{R}(K)=2 D_{\mu} v^{\mu}-v^{2}-K_{\sigma}^{\mu}{ }_{\nu} K_{\nu \mu}^{\sigma},
\end{gathered}
$$

$R^{\mu}{ }_{\nu \lambda \rho}(\Gamma)$ is the Riemann curvature constructed out of the Christoffel symbol $\Gamma_{\nu \lambda}^{\mu}$ and $\mathcal{P}_{\mu \nu \lambda}=$ $L_{\mu \nu \lambda}-L_{\lambda \mu \nu}-L_{\nu \mu \lambda}$. They satisfy the following symmetries and identities:

Case I: $\mathcal{R}^{\mu}{ }_{\nu \lambda \rho}(\Gamma, M)$

$$
\begin{aligned}
\mathcal{R}^{\mu}{ }_{\nu \lambda \rho} & =-\mathcal{R}^{\mu}{ }_{\nu \rho \lambda} \\
\mathcal{R}^{\mu}{ }_{\{\nu \lambda \rho\}} & =0 \\
\mathcal{D}_{\sigma} \mathcal{R}_{\mu \nu \lambda \rho}+\mathcal{D}_{\rho} \mathcal{R}_{\mu \nu \sigma \lambda}+\mathcal{D}_{\lambda} \mathcal{R}_{\mu \nu \rho \sigma} & =0 \\
\mathcal{D}_{\{\mu} \mathcal{H}_{\nu \lambda\}} & =0 \\
\mathcal{R}_{\nu \lambda}-\mathcal{R}_{\lambda \nu} & =2 \mathcal{H}_{\lambda \nu}
\end{aligned}
$$

where $\mathcal{H}_{\nu \lambda}=\partial_{\nu} w_{\lambda}-\partial_{\lambda} w_{\nu}$ and $\{\mu \nu \lambda\}$ denotes cyclic permutation of the indices.

Case II: $\mathcal{R}^{\mu}{ }_{\nu \lambda \rho}(K)$

$$
\begin{gathered}
\mathcal{R}^{\mu}{ }_{\nu \lambda \rho}=-\mathcal{R}^{\mu}{ }_{\nu \rho \lambda} \\
\mathcal{R}_{\nu \lambda}-\mathcal{R}_{\lambda \nu}=2\left[\mathcal{H}_{[\nu \lambda]}-D_{\mu} K^{\mu}{ }_{[\nu \lambda]}+v_{\sigma} K^{\sigma}{ }_{[\nu \lambda]}+K_{\sigma[\lambda}^{\mu} K_{\nu] \mu}^{\sigma}\right] .
\end{gathered}
$$

In $\left(W_{n}, g\right)$ space-time the Ricci tensor is:

$$
\mathcal{R}_{\mu \nu}(\Gamma, M)=R_{\mu \nu}(\Gamma)+\left[g_{\mu \nu} D^{\alpha} w_{\alpha}+(n-2) D_{\nu} w_{\mu}\right]-\frac{1}{4}(n-2)\left(g_{\mu \nu} w^{2}-w_{\mu} w_{\nu}\right)-\frac{1}{2} \mathcal{H}_{\mu \nu}
$$

while the Ricci scalar is given by:

$$
\mathcal{R}(\Gamma, M)=R(\Gamma)+(n-1) D^{\alpha} w_{\alpha}-\frac{1}{4}(n-1)(n-2) w^{2} .
$$


Consider manifolds with Euclidean signature and hermitian Dirac matrices satisfying:

$$
\left\{\gamma^{a}, \gamma^{b}\right\}=2 \delta^{a b},
$$

$\left(\gamma^{a}\right)^{2}=I$ and $\left(\gamma^{a}\right)^{\dagger}=\gamma^{a}$, where $a=1, \cdots, 5$. In this representation we make use of the following gamma matrix identities:

$$
\begin{gathered}
\gamma_{a} \gamma_{b} \gamma_{c}=\gamma_{a} \delta_{b c}-\gamma_{b} \delta_{a c}+\gamma_{c} \delta_{a b}+\epsilon_{a b c d} \gamma_{5} \gamma_{d} \\
{\left[\gamma_{a}, \gamma_{b}\right]=-\epsilon_{a b c d} \gamma_{5} \gamma^{c d} .}
\end{gathered}
$$

\section{References}

[1] C. G. Callan, S. Coleman and R. Jackiw, Ann. of Phys. 59 (1970) 42.

[2] E. S. Fradkin and G. A. Vilkovisky, Phys. Lett. A77 (1978) 262.

[3] D. Hochberg and G. Plunien, Phys. Rev. D43 (1991) 3358.

[4] I. L. Buchbinder and I. L. Shapiro, Phys. Lett. B151 (1985) 263;

G. Cognola and S. Zerbini, Phys. Lett. B214 (1988) 70;

R. Hammond, Phys. Rev. D52 (1995) 6918;

A. Dobado and A. Maroto, Mod. Phys. Lett. A12 (1997) 3003;

C. Lammerzahl, Phys. Lett. A228 (1997) 223.

[5] A. S. Belyaev and I. L. Shapiro, Nucl. Phys. B543 (20) $1999 .$.

[6] E. Braaten, T. L. Curtright and C. K. Zachos, Nucl. Phys. B260 (1985) 630.

[7] F. Hehl, P. Von der Heyde, G. Kerlick, Rev. Mod. Phys. 48 (1971) 393;

F. Hehl, J. McCrea, E. Mielke, Y. Ne'eman, Phys. Rep. 258 (1995) 1.

[8] K. Hayashi and A. Bregman, Ann. of Phys. 75 (1973) 562;

K. Hayashi and T. Shirafuji, Phys. Rev. D19 (1979) 3524;

K. Hayashi, K. Nomura and T. Shirafuji, Prog. Theor. Phys. 84 (1990) 1085.

[9] J. D. McCrea, Class. Quantum Grav. 9 (1992) 553.

[10] S. Browne and D. Šijački, Ann. of Phys. 99 (1976) 92 ;

D. Šijački, Frontiers in Particle Physics 83, World Scientific.

[11] I. L. Buchdinder, S. D. Odintsov and S. D. Shapiro: "Effective action in quantum gravity" (IOP Bristol and Philadelphia 1992).

[12] H. T. Nieh and M. L. Yan, J. Math. Phys. 23 (1982) 373. 